\title{
Some Results about Duality of Generalized Bessel Sequences
}

\author{
Mohammad Sadegh Asgari* and Golsa Kavian
}

Department of Mathematics, Faculty of Science, Islamic Azad University, Central Tehran Branch, Tehran, Iran; moh.asgari@iauctb.ac.ir, kaviangolsa@yahoo.com

\section{Abstract}

Bessel sequences in Hilbert spaces have been studied intensively for the past years. In this paper we show that in any Hilbert space, any pairs of g-Bessel sequences can be extended to a pair of dual g-frames. We also analyze the dual, pseudodual and approximate dual of frames and fusion frames. We generalize the restricted isometry property for $g$-frames. We also discuss the stability of $g$-frames under Erasure of operators.

Keywords: Approximate Dual Frames, Bessel Sequences, Dual Frames, Fusion Frames, G-frames, Pseudo-dual Frames

\section{Introduction}

Let $\mathrm{H}$ denote a separable Hilbert space. A sequence $\left\{f_{i}\right\}_{i \in I}$ in $\mathrm{H}$ is called a frame if there exist constants $0<A \leq B<\infty$ such that

$$
A\|f\|^{2} \leq \sum_{i \in I}\left|\left\langle f, f_{i}\right\rangle\right|^{2} \leq B\|f\|^{2} \quad \forall f \in H
$$

The sequence $\left\{f_{i}\right\}_{i \in I}$ is a Bessel sequence if at least the upper bound in (1) is satisfied. The number $B$ is called an upper frame bound, and the smallest possible value for $B$ is called the optimal bound. A frame is tight if we can choose $A=B$ in (1) and if $A=B=1$, it is a Parseval frame. If $\left\|f_{i}\right\|=c$ for all $i \in I$ this is an equal norm frame, and if $c=1$ it is a unit norm frame. For any frame $\left\{f_{i}\right\}_{i \in I}$ there exists at least one dual frame, i.e., a frame $\left\{g_{i}\right\}_{i \in I}$ for which $f=\sum_{i \in I}\left\langle f, g_{i}\right\rangle f_{i}$ for all $f \in H$. If $\left\{f_{i}\right\}_{i \in I}$ is a Bessel sequence with bound $B<1$, how can we find two sequences $\left\{g_{i}\right\}_{i \in I}$ and $\left\{p_{i}\right\}_{i \in I}$ such that $\left\{f_{i}+g_{i}\right\}_{i \in I}$ and $\left\{p_{i}\right\}_{i \in I}$ are dual frames, i.e., such that

$$
f=\sum_{i \in I}\left\langle f, p_{i}\right\rangle\left(f_{i}+g_{i}\right)=\sum_{i \in I}\left\langle f, f_{i}+g_{i}\right\rangle p_{i}
$$

In this paper we aim at the more general results of the type in (2). For any Bessel sequence $F=\left\{f_{i}\right\}_{i \in I}$ the synthesis operator is the bounded linear operator $T_{F}: l^{2}(I) \rightarrow H$, given by $T_{F}\left(\left\{c_{i}\right\}_{i \in I}\right)=\sum_{i \in I} c_{i} f_{i}$.

*Author for correspondence
The analysis operator for $F$ is $T_{F}^{*}$ and is given by $T_{F}^{*} f=\left\{\left\langle f, f_{i}\right\rangle\right\}_{i \in I^{*}}$. The frame operator is the positive self-adjoint invertible operator $S_{F}=T_{F} T_{F}^{*}$ and satisfies $S_{F} f=\sum_{i \in I}\left\langle f, f_{i}\right\rangle f_{i}$. Reconstruction is given by

$$
f=\sum_{i \in I}\left\langle f, f_{i}\right\rangle S_{F}^{-1} f_{i}=\sum_{i \in I}\left\langle f, S_{F}^{-\frac{1}{2}} f_{i}\right\rangle S_{F}^{-\frac{1}{2}} f_{i} \quad \forall f \in H
$$

In particular, $\left\{S_{F}^{-\frac{1}{2}} f_{i}\right\}_{i \in I}$ is a Parseval frame for $H$. Frames were first introduced in 1952 by Duffin and Schaeffer ${ }^{1}$ in the study of nonharmonic Fourier series, reintroduced in 1986 by Daubechies, Grossman and Meyer $^{2}$. Fusion frames for Hilbert spaces first formally were defined by Casazza and Kutyniok ${ }^{3}$ and g-frames also were introduced by Sun ${ }^{4}$.

Let $H, K^{\prime}$ be two separable Hilbert spaces and $\left\{W_{i}\right\}_{i \in I}$ be a sequence of closed subspaces of $K$, where $I$ is a subset of $\mathbb{Z}$. Let $\mathfrak{E}\left(H, W_{i}\right)$ be the collection of all bounded linear operators from $H$ into $W_{i}$ Recall that a family of operators $\Lambda=\left\{\Lambda_{i} \in \mathfrak{E}\left(H, W_{i}\right): i \in I\right\}$ is said to be a generalized frame, or simply a g-frame for $\mathcal{H}$ with respect to $\left\{W_{i}\right\}_{i \in I}$ if there exist constants $0<C \leq D<\infty$ such that

$$
C\|f\|^{2} \leq \sum_{i \in I}\left\|\Lambda_{i} f\right\|^{2} \leq D\|f\|^{2} \quad \forall f \in H
$$

The constants $C$ and $D$ are called g-frame bounds and $\sup p_{i \in I} \operatorname{rank} \Lambda_{i}$ is called the multiplicity of the g-frame. We 
call $\Lambda$ a tight g-frame if $C=D$ and it is a Parseval g-frame if $C=D=1 . \Lambda$ is called a $\varepsilon$-g-frame for $H$ if $C=\frac{1}{1+\varepsilon}$ and $D=1+\varepsilon$ for some $\varepsilon>0$. If the right-hand side of (4) holds, then $\Lambda$ is said a g-Bessel sequence for $H$ with respect to $\left\{W_{i}\right\}_{i \in I}$. The representation space associated with a $\mathrm{g}$ Bessel sequence $\Lambda=\left\{\Lambda_{i}\right\}_{i \in I}$ is defined by

$$
\left(\sum_{i \in I} \oplus W_{i}\right)_{l^{2}}=\left\{\left\{g_{i}\right\}_{i \in I}: g_{i} \in W_{i} \text {, and } \sum_{i \in I}\left\|g_{i}\right\|^{2}<\infty\right\}
$$

The synthesis operator of $\Lambda$ given by

$$
T_{A}:\left(\sum_{i \in I} \oplus W_{i}\right)_{l^{2}} \rightarrow H \quad T_{A}\left(\left\{g_{i}\right\}_{i \in I}\right)=\sum_{i \in I} \Lambda_{i}^{*} g_{i}
$$

The adjoint operator of $T_{A}^{*}$, which is called the analysis operator also obtain as follows

$$
T_{\Lambda}^{*}: H \rightarrow\left(\sum_{i \in I} \oplus W_{i}\right)_{l^{2}} \quad T_{\Lambda}^{*} f=\left\{\Lambda_{i} f\right\}_{i \in I}
$$

By composing $T_{A}$ with its adjoint $T_{A}^{*}$, we obtain the fusion frame operator

$$
S_{\Lambda}: H \rightarrow H \quad S_{A} f=T_{A} T_{\Lambda}^{*} f=\sum_{i \in I} \Lambda_{i}^{*} \Lambda_{i} f,
$$

which is a bounded, self-adjoint, positive and invertible operator and $C I_{H} \leq S_{A} \leq D I_{H}$. The canonical dual g-frame for $\left\{\Lambda_{i}\right\}_{i \in I}$ is defined by $\left\{\tilde{\Lambda}_{i}\right\}_{i \in I}$ where $\tilde{\Lambda}_{i}=\Lambda_{i} S_{\Lambda}^{-1}$, which is also a $g$-frame for $H$ with $g$-frame bounds $\frac{1}{D}$ and $\frac{1}{C}$, respectively. Also we have

$$
f=\sum_{i \in I} \Lambda_{i}^{*} \tilde{\Lambda}_{i} f=\sum_{i \in I} \tilde{\Lambda}_{i}^{*} \Lambda_{i} f \quad \forall f \in H
$$

For more details about the theory and applications of frames, please refer ${ }^{1,2,5,6}$ and about fusion frames refer ${ }^{3,7,8}$, about g-frames refer ${ }^{4,9,10}$.

The main subject of this paper deals with duality of Bessel sequences and g-Bessel sequences and constructs fusion frames by $\mathrm{g}$-frames with restricted isometry property.

The paper is organized as follows: Section 2, contains an extension of g- Bessel and g-Bessel sequences to dual frames and dual g-frames. In this section we show that the sum of any Bessel sequence with Bessel bound less than one with a Parseval frame is a frame and a Bessel sequence is an inner summand of a frame. Next we develop this result to the $\mathrm{g}$-frame situation. In Section 3 , we consider the dual, pseudo-dual and approximate dual frames, fusion frames and we obtain several characterizations of all this dual frames. In Section 4, we generalize the restricted isometry property to the g-frame situation. In Section 5, we study the conditions which under removing some element from a g-frame, again we obtain another g-frame.

\section{Duality of Bessel and g-Bessel Sequences}

Li and Sun ${ }^{10}$ expanded every Bessel sequence to a tight frame by adding some elements. In this section we show that the sum of any Bessel sequence with Bessel bound less than one with a Parseval frame is a frame for $H$ and we prove that a Bessel sequence is an inner summand of a frame.

Let $F=\left\{f_{i}\right\}_{i \in I}$ and $G=\left\{g_{i}\right\}_{i \in I}$ be two Bessel sequences for $H$ with synthesis operators $T_{F}$ and $T_{G}$ respectively. Then we say that $F$ and $G$ are dual frames for $H$ if $T_{F} T_{G}^{*}=I_{H}$ or $T_{G} T_{F}^{*}=I_{H}$, i.e.

$$
f=\sum_{i \in I}\left\langle f, g_{i}\right\rangle f_{i}=\sum_{i \in I}\left\langle f, f_{i}\right\rangle g_{i}, \quad \forall f \in H
$$

Notation 2.1 For each index set $I$, we define the space $l(I, H)$ by

$$
l(I, H)=\left\{\left\{f_{i}\right\}_{i \in I}: f_{i} \in H, \text { and } \sup _{i \in I}\left\|f_{i}\right\|<\infty\right\} .
$$

It is easy to check that $l(I, H)$ with the point-wise operations and norm defined by

$$
\left\|\left\{f_{i}\right\}_{i \in I}\right\|=\sup _{i \in I}\left\|f_{i}\right\|
$$

is a Banach space. Let $B(I, H)$ be the set of all Bessel sequences, $F(I, H)$ be the collection of all frames and $P(I$, $H$ ) denote the set of all Parseval frames indexed by $I$ for $H$ respectively. Since for any $F=\left\{f_{i}\right\}_{i \in I} \in B(I, H)$ there exists an operator

$T \in \mathcal{E}\left(l^{2}(I), H\right)$ such that $\mathrm{T} \delta_{i}=f_{i}$ for all $i \in I$, where $\left\{\delta_{i}\right\}_{i \in I}$ is an orthonormal basis of $l^{2}(I)$. Thus $B(I, H)$ is a subspace of $l(I, H)$ and

$$
P(I, H) \subseteq F(I, H) \subseteq B(I, H) \subseteq l(I, H)
$$

The following theorem shows that the sum of any Bessel sequence with Bessel bound less than one with a Parseval frame is a frame for $H$.

TheOrem 2.2 Let $F=\left\{f_{i}\right\}_{i \in I} \in B(I, H)$ with Bessel bound $B<1$. Then

$$
F+P(I, H) \subseteq F(I, H)
$$


Proof. Let $G=\left\{g_{i}\right\}_{i \in I} \in P(I, H)$. Then for all $f \in H$ we have

$$
\begin{aligned}
\left\|T_{F} T_{G}^{*} f\right\|^{2} & =\sup _{\|g\|=1}\left|\left\langle T_{F} T_{G}^{*} f, g\right\rangle\right|^{2} \\
& =\sup _{\|g\|=1}\left|\sum_{i \in I}\left\langle f, g_{i}\right\rangle\left\langle f_{i}, g\right\rangle\right|^{2} \\
& \leq \sup _{\|g\|=1} \sum_{i \in I}\left|\left\langle f, g_{i}\right\rangle\right|^{2} \sum_{i \in I}\left|\left\langle g, f_{i}\right\rangle\right|^{2} \leq B\|f\|^{2}
\end{aligned}
$$

Thus $\left\|T_{F} T_{G}^{*}\right\| \leq \sqrt{B}<1$, and so $I_{H}+T_{F} T_{G}^{*}$ an invertible operator in $\mathfrak{E}(H)$. If we set $\Theta=\left(I_{H}+T_{F} T_{G}^{*}\right)^{-1}$ and $U=F+G$ with $U=\left\{u_{i}\right\}_{i \in I}$. Then we compute

$$
\begin{aligned}
f=\left(I_{H}+T_{F} T_{G}^{*}\right) \Theta f & =\sum_{i \in I}\left\langle\Theta f, g_{i}\right\rangle f_{i}+\sum_{i \in I}\left\langle\Theta f, g_{i}\right\rangle g_{i} \\
& =\sum_{i \in I}\left\langle f, \Theta^{*} g_{i}\right\rangle u_{i},
\end{aligned}
$$

for all $f \in H$. This shows that $U \in F(I, H)$ with frame bounds $\|\Theta\|^{-2}$ and $(1+\sqrt{B})^{2}$.

The next result shows that a Bessel sequence is an inner summand of a frame.

Corollary 2.3 Let $F \in B(I, H)$ be a Bessel sequence. Then there exists a tight frame $G \in F(I, H)$ such that $F+G \in F(I, H)$.

Proof. Let $B$ be the Bessel bound for $F=\left\{f_{i}\right\}_{i \in I}$, then $\left\{\frac{1}{\sqrt{2 B}} f_{i}\right\}_{i \in I}$ is a Bessel sequence with the Bessel bound less than one. By Theorem $2.2\left\{\frac{1}{\sqrt{2 B}} f_{i}+e_{i}\right\}_{i \in I}$ is a frame for $H$, where $\left\{e_{i}\right\}_{i \in I}$ is an arbitrary Parseval frame. Define $g_{i}=\sqrt{2 B} e_{i}$ for all $i \in I$, then $G=\left\{g_{i}\right\}_{i \in I}$ is a tight frame and $F+G=\left\{\sqrt{2 B\left(\frac{1}{\sqrt{2 B}} f_{i}+e_{i}\right)}\right\}_{i \in I}$ is also a frame for $H$. The next theorem changes every Bessel sequence to a dual frame by summing it with any Parseval frame.

Theorem 2.4 Let $F \in B(I, H)$ with Bessel bound $B<1$ and let $\varepsilon \in P(I, H)$ Then there exists a $G \in B(I, H)$ such that $F+\varepsilon$ and $G+\varepsilon$ are dual frames.

Proof. Let $F=\left\{f_{i}\right\}_{i \in I}$ and $\varepsilon=\left\{e_{i}\right\}_{i \in I}$. Since $B<1$, hence $I_{H}+T_{F} T_{\varepsilon}^{*}$ is an invertible operator in $\mathfrak{E}(H)$. If we define $\Theta=-\left(I_{H}+T_{F} T_{\varepsilon}^{*}\right)^{-1} T_{F} T_{\delta}^{*}$ and $g_{i}=\Theta^{*} e_{i}$ for all $i \in I$. Then $G=\left\{g_{i}\right\}_{i \in I}$ is a Bessel sequence for $H$ and for all $f \in H$ we have

$$
\begin{aligned}
f= & \left(I_{H}+T_{F} T_{\varepsilon}^{*}\right) \Theta f+T_{\varepsilon} T_{\varepsilon}^{*} f+T_{F} T_{\varepsilon}^{*} f \\
= & T_{\varepsilon} T_{\varepsilon}^{*} \Theta f+T_{\varepsilon} T_{\varepsilon}^{*} f+T_{F} T_{\varepsilon}^{*} \Theta f+T_{F} T_{\varepsilon}^{*} f \\
= & \sum_{i \in I}\left\langle\Theta f, e_{i}\right\rangle e_{i}+\sum_{i \in I}\left\langle f, e_{i}\right\rangle e_{i} \\
& +\sum_{i \in I}\left\langle\Theta f, e_{i}\right\rangle f_{i}+\sum_{i \in I}\left\langle f, e_{i}\right\rangle f_{i} \\
= & \sum_{i \in I}\left\langle f, g_{i}+e_{i}\right\rangle\left(f_{i}+e_{i}\right),
\end{aligned}
$$

This finishes the proof.

Corollary 2.5 Let $F \in B(I, H)$ with Bessel bound $B<$ 1 and let $\varepsilon \in P(I, H)$. Then there exists a $G \in B(I, H)$ such that $F+\varepsilon$ and $G$ are dual frames.

Proof. Suppose that $F=\left\{f_{i}\right\}_{i \in I}$ and $\varepsilon=\left\{e_{i}\right\}_{i \in I}$ Since $I_{H}+T_{F} T_{\varepsilon}^{*}$ is invertible on $H$, Thus if weset $\Theta=\left(I_{H}+T_{F} T_{\varepsilon}^{*}\right)^{-1}$ and $g_{i}=\Theta^{*} e_{i}$ for all $i \in I$. Then for all $f \in H$ we have

$$
\begin{aligned}
f & =\left(I_{H}+T_{F} T_{\varepsilon}^{*}\right) \Theta f=T_{\varepsilon} T_{\varepsilon}^{*} \Theta f+T_{F} T_{\varepsilon}^{*} \Theta f \\
& =\sum_{i \in I}\left\langle\Theta f, e_{i}\right\rangle e_{i}+\sum_{i \in I}\left\langle\Theta f, e_{i}\right\rangle f_{i} \\
& =\sum_{i \in I}\left\langle f, g_{i}\right\rangle\left(f_{i}+e_{i}\right) .
\end{aligned}
$$

From this completes the proof.

Corollary 2.6 For every $F \in B(I, H)$ there exist $G \in B(I, H)$ and a tight frame $U \in F(I, H)$ such that $F+U$ and $G$ are dual frames for $H$.

Proof. Let $B$ be the Bessel bound of $F=\left\{f_{i}\right\}_{i \in I}$ and let $\left\{e_{i}\right\}_{i \in I}$ denote any Parseval frame for $H$. By Theorem 2.4 there exists a Bessel sequence $\left\{v_{i}\right\}_{i \in I}$ for $H$ such that $\left\{\frac{1}{\sqrt{2 B}} f_{i}+e_{i}\right\}_{i \in I}$ and $\left\{v_{i}+e_{i}\right\}_{i \in I}$ are dual frames for $H$. Put $G=\left\{g_{i}\right\}_{i \in I}, \quad U=\left\{u_{i}\right\}_{i \in I}$ with $g_{i}=\frac{1}{\sqrt{2 B}} v_{i}+\frac{1}{\sqrt{2 B}} e_{i}$ and $u_{i}=\sqrt{2 B} e_{i}$ for all $i \in I$. Then for all $f \in H$ we have

$$
\begin{aligned}
\sum_{i \in I}\left\langle f, g_{i}\right\rangle\left(f_{i}+u_{i}\right) & =\sum_{i \in I}\left\langle f, \frac{1}{\sqrt{2 B}} v_{i}+\frac{1}{\sqrt{2 B}} e_{i}\right\rangle\left(f_{i}+\sqrt{2 B} e_{i}\right) \\
& =\sum_{i \in I}\left\langle f, v_{i}+e_{i}\right\rangle\left(\frac{1}{\sqrt{2 B}} f_{i}+e_{i}\right)=f
\end{aligned}
$$

From this the claim follows immediately.

In the following theorem we show that every Bessel sequence can be expanded to a dual frame by adding it to a Parseval frame. Another form of this result can be found ${ }^{10}$ in Corollary 3.2. 
Theorem 2.7 Let $F=\left\{f_{i}\right\}_{i \in I}$ be a Bessel sequence with Bessel bound $B$ and $\varepsilon=\left\{e_{i}\right\}_{i \in I}$ be a Parseval frame for $H$. Then for all $\alpha>B$, there exists a Bessel sequence $\left\{g_{i}\right\}_{i \in i}$ for $H$ such that $\left\{f_{i}, e_{i}\right\}_{i \in I}$ and $\left\{\frac{1}{\alpha} f_{i}, g_{i}\right\}_{i \in I}$ are dual frames for $H$.

Proof. Since $\alpha>B$, hence $\Theta=I_{H}+\frac{1}{\alpha} T_{F} T_{F}^{*}$ is a linear bounded and positive operator on $H$. Thus if we define $g_{i}=\Theta^{*} e_{i}$ for all $i \in I$. Then $\left\{g_{i}\right\}_{i \in I}$ is a Bessel sequence for $H$ and for all $f \in H$ we have

$$
\begin{aligned}
\sum_{i \in I}\left\langle f, \frac{1}{\alpha} f_{i}\right\rangle f_{i}+\sum_{i \in I}\left\langle f, g_{i}\right\rangle e_{i} & \\
& =\sum_{i \in I}\left\langle f, \frac{1}{\alpha} f_{i}\right\rangle f_{i}+\sum_{i \in I}\left\langle\Theta f, e_{i}\right\rangle e_{i} \\
& =\frac{1}{\alpha} T_{F} T_{F}^{*} f+\Theta f=f
\end{aligned}
$$

This finishes the proof.

Let $\Lambda=\left\{\Lambda_{i}\right\}_{i \in I}$ and $\Gamma=\left\{\Gamma_{i}\right\}_{i \in I}$ be g-Bessel sequences for $H$ with synthesis operators $T_{\Lambda}$ and $T_{\Gamma}$ respectively. Then we say that $\Lambda$ and $\Gamma$ are dual g-frames for $H$ if $T_{\Lambda} T_{\Gamma}^{*}=I_{H}$ or $T_{\Gamma} T_{\Lambda}^{*}=I_{H}$. In the following we show that any pair of g-Bessel sequences can be extended to pair of dual g-frames. This result, generalizes a result of Christensen, Oh Kim and Young $\mathrm{Kim}^{6}$ to the situation of g-frames.

Theorem 2.8 Let $\Lambda=\left\{\Lambda_{i}\right\}_{i \in I}$ and $\Gamma=\left\{\Gamma_{i}\right\}_{i \in I}$ be two g-Bessel sequences for $H$ with respect to $\left\{W_{i}\right\}_{i \in I}$. Then there exist g-Bessel sequences $\left\{E_{j}\right\}_{j \in J}$ and $\left\{\Omega_{j}\right\}_{j \in J}$ for $H$ with respect to $\left\{\mathrm{V}_{j}\right\}_{j \in J}$, such that $\left\{\Lambda_{i}\right\}_{i \in I} \cup\left\{E_{j}\right\}_{i \in J}$ and $\left\{\Gamma_{i}\right\}_{i \in I} \cup\left\{\Omega_{j}\right\}_{j \in J}$ form a pair of dual g-frames for $H$ with respect to $\left\{W_{i}\right\}_{i \in I} \cup\left\{V_{j}\right\}_{j \in j}$.

Proof. Assume that $\left\{\varphi_{j}\right\}_{j \in J}$ and $\left\{\psi_{j}\right\}_{j \in J}$ are any pair of dual g-frames for $H$ respect to $\left\{V_{j}\right\}_{j \in J}$ and let $\Theta=I_{H}-T_{\Lambda} T_{\Gamma}^{*}$. Then for any $f \in H$ we have

$$
f=\Theta f+T_{\Gamma} T_{\Lambda}^{*} f=\sum_{j \in J} \psi_{j}^{*} \varphi_{j} \Theta f+\sum_{j \in J} \Gamma_{i}^{*} \Lambda_{i} f
$$

If we set $E_{j}=\Phi_{j} \Theta$ and $\Omega_{j}=\Psi_{j}$ for all $j \in J$. Then $\left\{\Lambda_{i}\right\}_{i \in I} \cup\left\{E_{j}\right\}_{j \in J}$ and $\left\{\Gamma_{i}\right\}_{i \in I} \cup\left\{\Omega_{j}\right\}_{j \in J}$ are dual g-frames for $H$ with respect to $\left\{W_{i}\right\}_{i \in I} \cup\left\{V_{j}\right\}_{j \in J}$.
The following corollaries are generalizations of the above results to the g-frames situation. We leave the proofs to interested readers.

Corollary 2.9 Let $\Lambda=\left\{\Lambda_{i}\right\}_{i \in I}$ be a g-Bessel sequence for $\mathrm{H}$ with respect to $\left\{W_{i}\right\}_{i \in I}$ with g-Bessel bound $B<1$. Then there exists g-Bessel sequence $\left\{\Gamma_{i}\right\}_{i \in I}$ for $H$ with respect to $\left\{W_{i}\right\}_{i \in I}$, such that $\left\{E_{i}+\Lambda_{i}\right\}_{i \in I}$ and $\left\{E_{i}+\Gamma_{i}\right\}_{i \in I}$ are dual g-frames for $H$ with respect to $\left\{W_{i}\right\}_{i \in I}$, where $\left\{E_{i}\right\}_{i \in I}$ is a Parseval g-frame for $H$ with respect to $\left\{W_{i}\right\}_{i \in I^{*}}$

COROLlaRY 2.10 For every g-Bessel sequence $\Lambda=\left\{\Lambda_{i}\right\}_{i \in I}$ with Bessel bound $B<1$ and each Parseval g-frame $E=\left\{E_{i}\right\}_{i \in I}$ for $H$ with respect to $\left\{W_{i}\right\}_{i \in I}$, there exists g-Bessel sequence $\left\{\Gamma_{i}\right\}_{i \in I}$ for $H$ with respect to $\left\{W_{i}\right\}_{i \in I}$ such that $\left\{\Lambda_{i}+E_{i}\right\}_{i \in I}$ and $\left\{\Gamma_{i}\right\}_{i \in I}$ are dual g-frames for $H$ with respect to $\left\{W_{i}\right\}_{i \in I}$.

Corollary 2.11 For every g-Bessel sequence $\left\{\Lambda_{i}\right\}_{i \in I}$ for $H$ with respect to $\left\{W_{i}\right\}_{i \in I}$ there exist g-Bessel sequence $\left\{\Gamma_{i}\right\}_{i \in I}$ and a tight g-frame $\left\{E_{i}\right\}_{i \in I}$ for $H$ with respect to $\left\{W_{i}\right\}_{i \in I}$ such that $\left\{\Lambda_{i}+E_{i}\right\}_{i \in I}$ and $\left\{\Gamma_{i}\right\}_{i \in I}$ are dual g-frames for $H$ with respect to $\left\{W_{i}\right\}_{i \in I}$.

\section{Approximate Duality of Bessel Fusion Sequences}

In this section we develop the theory of duality of frames to the fusion frames situation.

Let $W=\left\{W_{i}\right\}_{i \in I}$ be a sequence of closed subspaces in $H$, and let $A=\left\{\alpha_{i}\right\}_{i \in l}$ be a family of weights, i.e., $\alpha_{i}>0$ for all $i \in I$. A sequence $W_{\alpha}=\left\{\left(W_{i}, \alpha_{i}\right)\right\}_{i \in I}$ is a fusion frame, if there exist real numbers $0<C \leq D<\infty$ such that,

$$
C\|f\|^{2} \leq \sum_{i \in I} \alpha_{i}^{2}\left\|\pi w_{i}(f)\right\|^{2} \leq D\|f\|^{2} \text { for all } f \in H,
$$

where, $\pi w_{i}$ is the orthogonal projection from $H$ onto $W_{i}$. The constant $C, D$ are called the fusion frame bounds. If the right-hand inequality of (6) holds, then we say that $W_{\alpha}$ is a Bessel fusion sequence with Bessel fusion bound $D$. Moreover if $F_{i}=\left\{f_{i j}\right\}_{j \in J_{i}}$ is a frame for $W_{i}$ for all $i \in I$. Then $W=\left\{W_{i}, \alpha_{i}, F_{i}\right\}_{i \in I}$ is called a fusion frame system for $H$. The constants $A, B$ are called the local frame bounds if they are the common frame bounds for the 
local frame $F_{i}=\left\{f_{i j}\right\}_{j \in J_{i}}$ for all $i \in I$. A collection of dual frames $G_{i}=\left\{g_{i j}\right\}_{j \in J_{i}}, i \in I$ associated with the local frames will be called local dual frames. By Theorem $3.2^{3}$, if $W=\left\{W_{i}, \alpha_{i}, F_{i}\right\}_{i \in I}$ is a fusion frame system for $\mathrm{H}$ with fusion frame bounds $C, D$ and local frame bounds $A, B$, then $F=\left\{\alpha_{i} f_{i j}\right\}_{i \in I, j \in J_{i}}$ is a frame for $H$ with frame bounds $A C$ and $B D$. Also if $F=\left\{\alpha_{i} f_{i j}\right\}_{i \in I, j \in J_{i}}$ is a frame for $H$ with frame bounds $C$ and $D$ then $W=\left\{W_{i}, \alpha_{i}, F_{i}\right\}_{i \in I}$ is a fusion frame system for $H$ with fusion frame bounds $\frac{C}{B}$ and $\frac{D}{A}$.

Definition3.1 Let $W_{\alpha}=\left\{\left(W_{i}, \alpha_{i}\right)\right\}_{i \in I}$ and $Z_{\beta}=\left\{\left(Z_{i}, \beta_{i}\right)\right\}_{i \in I}$ be Bessel fusion sequences for $H$ with synthesis operator $T_{w_{\alpha}}$ and $T_{Z_{\beta}}$ respectively. Then

(i) $W_{\alpha}, Z_{\beta}$ are called dual fusion frames for $H$ if $T_{w_{\alpha}} T_{Z_{\beta}}^{*}=I_{H}$ or $T_{Z_{\beta}} T_{w_{\alpha}}^{*}=I_{H}$.

(ii) $W_{\alpha}, Z_{\beta}$ are said to be approximate dual fusion frames for $H$ if $\left\|I_{H}-T_{w_{\alpha}} T_{Z_{\beta}}^{*}\right\|<1$ or $\left\|I_{H}-T_{Z_{\beta}} T_{w_{\alpha}}^{*}\right\|<1$.

(iii) $W_{\alpha}, Z_{\beta}$ are called pseudo-dual fusion frames for $H$ if $T_{w_{\alpha}} T_{Z_{\beta}}^{*}$ or $T_{Z_{\beta}} T_{w_{\alpha}}^{*}$, is a bijection on $H$.

Therom 3.2 For each $i \in I$ let $\alpha_{i}>0$ and $J_{i}=J_{i 1} \cup J_{i 2}$ be a partition of $J_{i}$ and let $W=\left\{\left(W_{i}, \alpha_{i},\left\{f_{i j}\right\}_{j \in J_{i 1}}\right)\right\}_{i \in I}$, $Z=\left\{\left(Z_{i}, \beta_{i},\left\{g_{i j}\right\}_{j \in J_{i 2}}\right)\right\}_{i \in I}$ be two fusion frame system for $H$. Define

$$
U_{i j}=\left\{\begin{array} { l l } 
{ \frac { 1 } { \sqrt { 2 } } f _ { i j } } & { j \in J _ { i 1 } } \\
{ \frac { 1 } { \sqrt { 2 } } \pi w _ { i } \tilde { g } _ { i j } } & { j \in J _ { i 2 } }
\end{array} \text { and } \quad V _ { i j } \left\{\begin{array}{ll}
\frac{1}{\sqrt{2}} g_{i j} & \in J_{i 2} \\
\frac{1}{\sqrt{2}} \pi_{Z_{i}} \tilde{f}_{i j} & j \in J_{i 1}
\end{array}\right.\right.
$$

for all $i \in I, j \in J_{i}$. Then the following conditions are equivalent:

(1) $W_{\alpha}=\left\{\left(W_{i}, \alpha_{i}\right)\right\}_{i \in I}$ and $Z_{\beta}=\left\{\left(Z_{i}, \beta_{i}\right)\right\}_{i \in I}$ are (dual, pseudo-dual, approximate dual) fusion frames.

(2) $\left\{\alpha_{i} U_{i j}\right\}_{i \in I, j \in J_{i}}$ and $\left\{\beta_{i} V_{i j}\right\}_{i \in I, j \in J_{i}}$ are (dual, pseudodual, approximate dual) frames for $H$.
Proof. This claim follows immediately from the fact that for $f \in H$ we have

$$
\begin{aligned}
& \sum_{i \in I} \sum_{j \in J_{i}}\left\langle f, \beta_{i} V_{i j}\right\rangle \alpha_{i} U_{i j} \\
= & \sum_{i \in I} \alpha_{i} \beta_{i} \sum_{j \in J_{i 1}}\left\langle f, V_{i j}\right\rangle U_{i j} \\
& +\sum_{i \in I} \alpha_{i} \beta_{i} \sum_{j \in J_{i 2}}\left\langle f, V_{i j}\right\rangle U_{i j} \\
= & \sum_{i \in I} \alpha_{i} \beta_{i} \sum_{j \in J_{i 1}}\left\langle f, \frac{1}{\sqrt{2}} \pi_{Z_{i}} \tilde{f}_{i j}\right\rangle \frac{1}{\sqrt{2}} f_{i j} \\
& +\sum_{i \in I} \alpha_{i} \beta_{i} \sum_{j \in I_{i 2}}\left\langle f, \frac{1}{\sqrt{2}} g_{i j}\right\rangle \frac{1}{\sqrt{2}} \pi_{w_{i}} \tilde{g}_{i j} \\
= & \sum_{i \in I} \frac{\alpha_{i} \beta_{i}}{2} \sum_{j \in J_{i 1}}\left\langle\pi_{Z_{i}}(f), \tilde{f}_{i j}\right\rangle f_{i j} \\
& +\sum_{i \in I} \frac{\alpha_{i} \beta_{i}}{2} \pi_{W_{1}}\left(\sum_{j \in I_{i 2}}\left\langle f, g_{i j}\right\rangle \tilde{g}_{i j}\right) \\
= & \sum_{i \in I} \alpha_{i} \beta_{i} \pi_{W_{1}} \pi_{Z_{1}}(f) .
\end{aligned}
$$

TheOrem 3.3 Let $W=\left\{W_{i}, \alpha_{i},\left\{f_{i j}\right\}_{j \in J_{i}}\right\}_{i \in I}$ be a fusion frame system and let $Z_{\beta}=\left\{\left(Z_{i}, \beta_{i}\right)\right\}_{i \in I}$ be a fusion Bessel sequence for $H$. Put $g_{i j}=\pi_{Z_{1}}\left(\tilde{f}_{i j}\right)$ for all $i \in I, j \in J i$. Then the following conditions are equivalent:

(1) $W_{\alpha}=\left\{\left(W_{i}, \alpha_{i}\right)\right\}_{i \in I}$ and $Z_{\beta}=\left\{\left(Z_{i}, \beta_{i}\right)\right\}_{i \in I}$ are (dual, pseudo-dual, approximate dual) fusion frames.

(2) $F=\left\{\alpha_{i} f_{i j}\right\}_{i \in I, j \in J_{i}}$ and $G=\left\{\beta_{i} g_{i j}\right\}_{i \in I, j \in J_{i}}$, are (dual, pseudo-dual, approximate dual) frames for $H$.

Proof. First we prove that $G$ is a Bessel sequence for $H$. Let $D$ be the Bessel fusion bound of $Z_{\beta}$ and $A, B$ be the local frame bounds of $W$, then for all $f \in H$ we have

$$
\begin{aligned}
\sum_{i \in I} \sum_{j \in J_{i}}\left|\left\langle f, \beta_{i} g_{i j}\right\rangle\right|^{2} & =\sum_{i \in I} \sum_{j \in J_{i}} \beta_{i}^{2}\left|\left\langle f, \pi_{Z i} \tilde{f}_{i j}\right\rangle\right|^{2} \\
& =\sum_{i \in I} \beta_{i}^{2} \sum_{j \in J_{i}}\left|\left\langle\pi_{Z_{i}}(f), \tilde{f}_{i j}\right\rangle\right|^{2} \\
& \leq \sum_{i \in I} \frac{\beta_{(i)}^{2}}{A}\left\|\pi_{\mathrm{w}_{i}} \pi_{Z_{i}}(f)\right\|^{2} \\
& \leq \frac{1}{A} \beta_{i}^{2} \sum_{i \in I}\left\|\pi_{Z_{i}}(f)\right\|^{2} \leq \frac{D}{A}\|f\|^{2} .
\end{aligned}
$$

Let $T_{F}$ and $T_{G}$ be the synthesis operators for $F$ and $G$ respectively. Then for all $F \in H$ we obtain 


$$
\begin{aligned}
T_{W_{\alpha}} T_{Z_{\beta}}^{*}(f) & =\sum_{i \in I} \alpha_{i} \beta_{i} \pi_{w_{1}} \pi_{Z_{1}}(f) \\
& =\sum_{i \in I} \alpha_{i} \beta_{i} \sum_{j \in J_{i}}\left\langle\pi_{Z_{1}}(f), \tilde{f}_{i j}\right\rangle f_{i j} \\
& =\sum_{i \in I} \sum_{j \in J_{i}}\left\langle f, \beta_{i} g_{i j}\right\rangle \alpha_{i} f_{i j}=T_{F} T_{G}^{*} f .
\end{aligned}
$$

This finishes the proof.

TheOREM 3.4Let $W_{\alpha}=\left\{\left(W_{i}, \alpha_{i}\right)\right\}_{i \in I}$ and $Z_{\beta}=\left\{\left(Z_{i}, \beta_{i}\right)\right\}_{i \in I}$ be Bessel fusion sequences for $H$ and let $T \in B(H)$ be abounded invertible operator such that $T^{*} T W_{i} \subseteq W_{i}, T^{*} T Z_{i} \subseteq Z_{i}$. Then

(1) $W_{\alpha}$ and $Z_{\beta}$ are (dual, pseudo-dual) fusion frames if and only if $T W_{\alpha}=\left\{\left(T W_{i}, \alpha_{i}\right)\right\}_{i \in I}$ and $T Z_{\beta}=\left\{\left(T Z_{i}, \beta_{i}\right)\right\}_{i \in I}$ are (dual, pseudo-dual) fusion frame for $H$.

(2) If $W_{\alpha}$ and $Z_{\beta}$ are approximate dual fusion frames and $\|T\|\left\|T^{-1}\right\|=1$ then $T W_{\alpha}=\left\{\left(T W_{i}, \alpha_{i}\right)\right\}_{i \in I}$ and $T Z_{\beta}=\left\{\left(T Z_{i}, \beta_{i}\right)\right\}_{i \in I}$ are also approximate dual fusion frames for $H$.

Proof. (1) Since $T$ is invertible and $T^{*} T W_{i} \subseteq W_{i}$. $T^{*} T Z_{i} \subseteq Z_{i}$ hence for all $i \in I$

$\pi_{T W_{i}}=T \pi_{W_{i}} T^{-1}, \quad \pi_{T Z_{i}}=T \pi_{Z_{i}} T^{-1}$. This implies that $T_{T W_{\alpha}} T_{T Z_{\beta}}^{*}=T T_{W_{\alpha}} T_{Z_{\beta}}^{*} T^{-1}$, that from this the claim follows immediately.

(2) We have $\left\|I d_{H}-T_{T W_{\alpha}} T_{T Z_{\beta}}^{*}\right\|=\left\|T T^{-1}-T T_{W_{a}} T_{Z_{\beta}}^{*} T^{-1}\right\| \leq \| I d_{H}$ $-T_{W_{\alpha}} T_{Z_{\beta}}^{*} \|$. From this the result follows at once.

Theorem 3.5 Let $W_{\alpha}=\left\{\left(W_{i}, \alpha_{i}\right)\right\}_{i \in I}$ be a fusion frame and let $Z_{\alpha}=\left\{\left(Z_{i}, \alpha_{i}\right)\right\}_{i \in I}$ be a Bessel fusion sequence for $H$. Suppose that $T: H \rightarrow H$ is a bounded invertible operator such that $T W_{i} \subseteq Z_{i}$ for all $i \in I$. Then $Z_{\alpha}=\left\{\left(Z_{i}, \alpha_{i}\right)\right\}_{i \in I}$ and $T W_{\alpha}=\left\{\left(T W_{i}, \alpha_{i}\right)\right\}_{i \in I}$ are pseudo-dual fusion frames for $H$. Moreover if $T W_{\alpha}$ is a Parseval fusion frame then $Z_{\alpha}$ and $T W_{\alpha}$ are dual fusion frames.

Proof. Since $T W_{i} \subseteq Z_{i}$ hence $\pi_{T W_{i}} \pi_{Z_{i}}=\pi_{Z_{i}} \pi_{T W_{i}}=\pi_{T W_{i}}$ for all $i \in I$. It follows that $T_{T W_{\alpha}} T_{Z_{\alpha}}^{*}=T_{Z_{\alpha}} T_{T W_{\alpha}}^{*}=S_{T W_{\alpha}}$. which finishes the proof.

Definition 3.6 Let $\left\{W_{i}\right\}_{i \in I}$ and $\left\{\tilde{W}_{i}\right\}_{i \in I}$ be closed subspaces in $H$ and $\varepsilon>0$. If for all $f \in H$ we have $\sum_{i \in I} \alpha_{i}^{2}\left\|\pi_{W_{i}}(f)-\pi_{\tilde{W}_{i}}(f)\right\|^{2} \leq \varepsilon\|f\|^{2}$.
Then we say that $\left\{\left(\tilde{W}_{i}, \alpha_{i}\right)\right\}_{i \in I}$ is a $\varepsilon$-perturbation of $\left\{\left(W_{i}, \alpha_{i}\right)\right\}_{i \in I^{\circ}}$

Theorem 3.7 Let $W_{\alpha}=\left\{\left(W_{i}, \alpha_{i}\right)\right\}_{i \in I}, Z_{\beta}=\left\{\left(Z_{i}, \beta_{i}\right)\right\}_{i \in I}$ be Bessel fusion sequences with Bessel fusion bounds $D_{1}, D_{2}$ respectively for $H$. Let $\tilde{W}_{\alpha}=\left\{\left(\tilde{W}_{i}, \alpha_{i}\right)\right\}_{i \in I}$ be a $\varepsilon$-perturbation of $W_{\alpha}$ and $\varepsilon D_{2}<1$. If $W_{\alpha}$ and $Z_{\beta}$ are dual fusion frames, then $\tilde{W}_{\alpha}$ and $Z_{\beta}$ are also approximate dual fusion frames for $H$.

Proof. By Proposition $2.47 \tilde{W}_{\alpha}$ is a Bessel fusion sequence for $H$. Now for all $f \in H$ we have

$$
\begin{aligned}
\left\|f-T_{\tilde{W}_{\alpha}} T_{Z_{\beta}}^{*}(f)\right\|^{2}= & \left\|T_{W_{\alpha}} T_{Z_{\beta}}^{*}(f)-T_{\tilde{W}_{\alpha}} T_{Z_{\beta}}^{*}(f)\right\|^{2} \\
& =\sup _{\|g\|=1}\left|\left\langle T_{W_{\alpha}} T_{Z_{\beta}}^{*}(f)-T_{\tilde{W}_{\alpha}} T_{Z_{\beta}}^{*}(f), g\right\rangle\right|^{2} \\
& \leq \sup _{\|g\|=1}\left(\sum_{i \in I} \alpha_{i} \beta_{i}\left\|\pi_{W_{i}}(f)-\pi_{\tilde{W}_{i}}(f)\right\|\left\|\pi_{Z_{i}}(g)\right\|\right)^{2} \\
& \leq \sup _{\|g\|=1} \sum_{i \in I} \alpha_{i}^{2}\left\|\pi_{W_{i}}(f)-\pi_{\tilde{W}_{i}}(f)\right\|^{2} \\
& \sum_{i \in I} \beta_{i}^{2}\left\|\pi_{Z_{i}}(g)\right\|^{2} \leq \varepsilon D_{2}\|f\|^{2}
\end{aligned}
$$

From this the result follows at once.

\section{The Restricted Isometry Property for g-frames}

In this section we generalize the restricted isometry property for g-frames. We denote $H_{N}$ for a Hilbert space with dimension $N$ and $\left\{e_{j}\right\}_{j=1}^{N}$ an orthonormal basis for $H_{N}$. Moreover, the Hilbert-Schmidt norm of operator $T \in L\left(H_{N}, K\right)$ is defined by $\|T\|_{H S}^{2}=\sum_{j=1}^{N}\left\|T e_{j}\right\|^{2}$.

Proposition 4.1 Let $\left\{\Lambda_{i}\right\}_{i \in I}$ be a g-Bessel sequence for $H$ with respect to $\left\{W_{i}\right\}_{i \in I}$. Then

(i) If $H$ is finite-dimensional, then $\left\{\left\|\Lambda_{i}\right\|_{H S}^{2}\right\}_{i \in I}$ is summable.

(ii) If $W$ is finite-dimensional and $\alpha=\left\|\Lambda_{i}\right\|=\| \Lambda_{j} \mid$ for all $i, j \in I$. Then $\left\{\Lambda_{i}\right\}_{i \in I}$ is a finite sequence.

(iii) If $H$ is finite-dimensional and $\Lambda=\left\{\Lambda_{t}\right\}_{i \in I}$ is a g-frame for $H$ with $g$-frame bounds $A$ and $B$. Then

$$
A \leq \frac{\sum_{i \in I}\left\|\Lambda_{i}\right\|_{H S}^{2}}{\operatorname{dim} H} \leq B .
$$


Proof. (i) Let $B$ be the g-Bessel bound for $\left\{\Lambda_{i}\right\}_{i \in I}$ and let $\left\{e_{j}\right\}_{j=1}^{N}$ be an orthonormal basis for $H$. Then we have

$$
\begin{aligned}
\sum_{i \in I}\left\|\Lambda_{i}\right\|_{H S}^{2} & =\sum_{i \in I} \sum_{j=1}^{N}\left\|\Lambda_{i} e_{j}\right\|^{2} \\
& =\sum_{i \in I}\left\|\Lambda_{i} e_{i}\right\|^{2} \leq B \sum_{j=1}^{N}\left\|e_{j}\right\|^{2}=B N .
\end{aligned}
$$

(ii) Since $\left\|\Lambda_{i}\right\|_{H S} \geq\left\|\Lambda_{i}\right\|=\alpha$ hence from the part (i) follows that $\left\{\Lambda_{i}\right\}_{i \in I}$ is finite.

(iii) Since $\sum_{i \in I}\left\|\Lambda_{i}\right\|_{H S}^{2}=\sum_{j=1}^{N}<S_{\Lambda} e_{j}, e_{\mathrm{j}}>$ and $A I_{H} \leq S_{\Lambda} \leq B I_{H}$ thus we obtain

$A \operatorname{dim} H=A \sum_{j=1}^{N}\left\|e_{j}\right\|^{2} \leq \sum_{j=1}^{N}\left\langle S_{\Lambda} \mathrm{e}_{\mathrm{j}}, \mathrm{e}_{\mathrm{j}}\right\rangle \leq B \sum_{J=1}^{N}\left\|e_{j}\right\|^{2}=B \operatorname{dim} H$

This yields $A \operatorname{dim} H \leq \sum_{i \in I}\left\|\Lambda_{i}\right\|_{H S}^{2} \leq B \operatorname{dim} H$.

From this the claim follows immediately.

Theorem 4.2 Let $A=\left\{\Lambda_{i}\right\}_{i=1}^{M}$ be a g-frame for $H_{N}$ with respect to $\left\{W_{i}\right\}_{i=1}^{M}$. Then

(i) The optimal bounds of $\Lambda$ are the smallest and biggest eigenvalues of g-frame operator $S_{\Lambda}$.

(ii) If $\left\{\lambda_{j}\right\}_{j=1}^{N}$ is a representation of eigenvalues of $S_{\Lambda}$. Then

$$
\sum_{j=1}^{N} \lambda_{j}=\sum_{i=1}^{M}\left\|\Lambda_{i}\right\|_{H S}^{2} \text { and } \lambda_{i}=\sum_{i=1}^{M}\left\|\Lambda_{i} e_{j}\right\|^{2},
$$

where, $\left\{\mathrm{e}_{\mathrm{j}}\right\}_{j=1}^{N}$ is the orthonormal basis consisting of eigenvectors of $S_{\Lambda}$.

Proof. To prove (i) see that since $S_{\Lambda}$ is a self-adjoint operator on $H_{N}$, thus $H_{N}$ has an orthonormal basis include eigenvectors of $S_{\Lambda}$. Let $\left\{\mathrm{e}_{j}\right\}_{j=1}^{N}$ be an orthogonal basis of $H_{N}$ include of eigenvectors of $S_{\Lambda}$. Let $\left\{\lambda_{j}\right\}_{i=1}^{N}$ be eigenvalues of $\left\{\mathrm{e}_{\mathrm{j}}\right\}_{j=1}^{N}$. Then for any $f \in H_{N}$ we have

$$
\begin{aligned}
\sum_{i=1}^{M}\left\|\Lambda_{i} f\right\|^{2}\left\langle S_{\Lambda} f, f\right\rangle & =\left\langle\sum_{j=1}^{N}\left\langle f, e_{j}\right\rangle S_{\Lambda} e_{j}, f\right\rangle \\
& =\sum_{J=1}^{N}\left\langle f, e_{j}\right\rangle\left\langle S_{\Lambda} e_{j}, f\right\rangle \\
& =\sum_{j=1}^{N}\left\langle f, e_{j}\right\rangle\left\langle\lambda_{j} e_{j}, f\right\rangle \\
& =\sum_{j=1}^{N} \lambda_{j}\left|\left\langle f, e_{j}\right\rangle\right|^{2} .
\end{aligned}
$$

Since for any $1 \leq i \leq N$ we have $\lambda_{\text {min }} \leq \lambda_{i} \leq \lambda_{\text {max }}$, thus

$$
\lambda_{\text {min }}\|f\|^{2} \leq \sum_{i=1}^{M}\left\|\Lambda_{i} f\right\|^{2} \leq \lambda_{\max }\|f\|^{2} .
$$

To prove (ii) we have:

$$
\begin{aligned}
\sum_{j=1}^{N} \lambda_{j} & =\sum_{j=1}^{N}\left\langle\lambda_{j} e_{j}, e_{j}\right\rangle=\sum_{j=1}^{N}\left\langle S_{\Lambda} e_{j}, e_{j}\right\rangle \\
& =\sum_{j=1}^{N} \sum_{i=1}^{M}\left\|\Lambda_{i} e_{j}\right\|^{2}=\sum_{i=1}^{M} \sum_{j=1}^{N}\left\|\Lambda_{i} e_{j}\right\|^{2} \\
& =\sum_{i=1}^{M}\left\|\Lambda_{i}\right\|_{H S}^{2} .
\end{aligned}
$$

Also we obtain $\sum_{i=1}^{M}\left\|\Lambda_{i} e_{j}\right\|^{2}=\left\langle S_{\Lambda} e_{i}, e_{i}\right\rangle=\left\langle\lambda_{j} e_{i}, e_{i}\right\rangle=\lambda_{j}$.

COROLlary 4.3 Let $\left\{\Lambda_{i}\right\}_{i=1}^{M}$ be a $A$-tight g-frame with unit Hilbert-Schmidt norm for $H_{N}$ with respect to $\left\{W_{i}\right\}_{i=1}^{M}$, then $A=\frac{M}{N}$.

Proof. This is a direct result from section (iii) in Proposition 4.1.

Definition 4.4 Let $\Lambda_{i} \in \mathfrak{E}\left(H, W_{i}\right)$ for all $i \in I$. Then

(i) $\left\{\Lambda_{i}\right\}_{i \in I}$ is called an orthonormal g-system for $\mathrm{H}$ with respect to $\left\{W_{i}\right\}_{i \in I}$, if

$$
\Lambda_{i} \Lambda_{j}^{*} g_{j}=g_{j} \delta_{i j} \text { for all } i, j \in I, g_{j} \in W_{j}
$$

(ii) If $H=\overline{\operatorname{span}}\left\{\Lambda_{i}^{*}\left(W_{i}\right)\right\}_{i \in I}$, then we say that $\left\{\Lambda_{i}\right\}_{i \in I}$ is g-complete.

(iii) We say that $\left\{\Lambda_{i}\right\}_{i \in I} g$-orthonormal basis for $H$ with respect to $\left\{W_{i}\right\}_{i \in I}$, if it is a g-orthonormal g-complete system for $H$ with respect to $\left\{W_{i}\right\}_{i \in I^{*}}$

(iv) $\left\{\Lambda_{i}\right\}_{i \in I}$ is g-complete and there are positive constants $A$ and $B$ such that for any finite subset $J \subseteq I$ and $g_{j} \in W_{j}$,

$$
A \sum_{j \in J}\left\|g_{j}\right\|^{2} \leq\left\|\sum_{j \in J} \Lambda_{j}^{*} g_{j}\right\|^{2} \leq B \sum_{j \in J}\left\|g_{j}\right\|^{2} \text {. }
$$

Then $\left\{\Lambda_{i}\right\}_{i \in I}$ is called a g-Riesz basis for $H$ with respect to $\left\{W_{i}\right\}_{i \in I}$. Moreover, $\left\{\Lambda_{i}\right\}_{i \in I}$ is called a $\varepsilon$-g-Riesz basis for $H$ if $A=\frac{1}{1+\varepsilon}$ and $B=1+\varepsilon$ for some $\varepsilon>0$. Also $\left\{\Lambda_{i}\right\}_{i \in I}$ is a $\varepsilon$-g-Riesz sequence if $\left\{\Lambda_{i}\right\}_{i \in I}$ is a $\varepsilon$-g-Riesz basis for $\overline{\operatorname{span}}\left\{\Lambda_{j}^{*}\left(W_{i}\right)\right\}_{i \in I}$.

The next proposition is similar to a result of Bodmann, Cahill and Casazza ${ }^{11}$ to the situation of g-frames. 
Proposition 4.5 Let $\left\{\Lambda_{i}\right\}_{i \in I}$ be a $\varepsilon$-g-Riesz sequence for $H$ with respect to $\left\{W_{i}\right\}_{i \in I}$ and let $\left\{I_{j}\right\}_{j=I}^{L}$ be a partition of $I$. Then for every $1 \leq j \leq L$ and for any sequence $\left\{g_{j k}\right\}_{k \in I_{j}} \in\left(\sum_{k \in I_{j}} \oplus W_{k}\right)_{l^{2}}$.

$$
\begin{aligned}
& \frac{1}{1+\varepsilon} \sum_{j=1}^{L}\left\|\sum_{k \in I_{j}} \Lambda_{k}^{*} g_{j k}\right\|^{2} \leq \sum_{j=1}^{L} \sum_{k \in I_{j}}\left\|g_{j k}\right\|^{2} \\
& \leq(1+\varepsilon) \sum_{j=1}^{L}\left\|\sum_{k \in I_{j}} \Lambda_{k}^{*} g_{j k}\right\|^{2} .
\end{aligned}
$$

Also

$$
\begin{aligned}
\frac{1}{(1+\varepsilon)^{2}} \sum_{j=1}^{L}\left\|\sum_{k \in I_{j}} \Lambda_{\mathrm{\kappa}}^{*} g_{j k}\right\|^{2} & \leq\left\|\sum_{j=1}^{L} \sum_{k \in I_{j}} \Lambda_{\mathrm{\kappa}}^{*} g_{j k}\right\|^{2} \\
& \leq(1+\varepsilon)^{2} \sum_{j=1}^{L}\left\|\sum_{k \in I_{j}} \Lambda_{\kappa}^{*} g_{j k}\right\|^{2} .
\end{aligned}
$$

Proof. For each $1 \leq j \leq L$ and for any sequence $\left\{g_{j k}\right\}_{k \in I_{j}} \in\left(\sum_{k \in I_{j}} \oplus W_{k}\right)_{l^{2}}$ we have

$$
\begin{aligned}
\frac{1}{1+\varepsilon} \sum_{j=1}^{L}\left\|\sum_{k \in I_{j}} \Lambda_{\kappa}^{*} g_{j k}\right\|^{2} & \leq \frac{1}{1+\varepsilon} \sum_{j=1}^{L}(1+\varepsilon) \sum_{k \in I_{j}}\left\|g_{j k}\right\|^{2} \\
& =\sum_{j=1}^{L} \sum_{k \in I_{j}}\left\|g_{j k}\right\|^{2} \leq \sum_{j=1}^{L}(1+\varepsilon)\left\|\sum_{k \in I_{j}} \Lambda_{\kappa}^{*} g_{j k}\right\|^{2} \\
& =(1+\varepsilon) \sum_{j=1}^{L}\left\|\sum_{k \in I_{j}} \Lambda_{\kappa}^{*} g_{j k}\right\|^{2}
\end{aligned}
$$

This yields

$$
\begin{aligned}
\frac{1}{1+\varepsilon} \sum_{j=1}^{L}\left\|\sum_{k \in I_{j}} \Lambda_{\mathrm{\kappa}}^{*} g_{j k}\right\|^{2} & \leq \frac{1}{1+\varepsilon} \sum_{j=1}^{L} \sum_{k \in I_{j}}\left\|g_{j k}\right\|^{2} \\
& \leq\left\|\sum_{j=1}^{L} \sum_{k \in I_{j}} \Lambda_{\mathrm{k}}^{*} g_{j k}\right\|^{2} \leq(1+\varepsilon) \sum_{j=1}^{L} \sum_{k \in I_{j}}\left\|g_{j k}\right\|^{2} \\
& \leq(1+\varepsilon)^{2} \sum_{j=1}^{L}\left\|\sum_{k \in I_{j}} \Lambda_{\mathrm{\kappa}}^{*} g_{j k}\right\|^{2} .
\end{aligned}
$$

It is known that if $\left\{\Lambda_{i}\right\}_{i \in I}$ is a g-Riesz basis for $H$ with respect to $\left\{W_{i}\right\}_{i \in I}$ with $\mathrm{g}$-Riesz constants $A$ and $B$, then $\left\{\Lambda_{i}\right\}_{i \in I}$ is a g-frame for $H$ with respect to $\left\{W_{i}\right\}_{i \in I}$ with same bounds $A$ and $B$. The next lemma is analogous to Lemma $3.3^{11}$ to the situation of $\mathrm{g}$-frames.

LEMMA 4.6 Let $\Lambda=\left\{\Lambda_{i}\right\}_{i \in I}$ be a $\varepsilon$-g-Riesz basis for $H$ with respect to $\left\{W_{i}\right\}_{i \in I}$. Then for all $n \in \mathrm{N}$ $\frac{1}{(1+\varepsilon)^{n}} I_{H} \leq S_{\Lambda}^{n} \leq(1+\varepsilon)^{n} I_{H}$ and $\frac{1}{(1+\varepsilon)^{n}} I_{H} \leq S_{\Lambda}^{-n} \leq(1+\varepsilon)^{n} I_{H}$.

Proof. Since $\left\{\Lambda_{i}\right\}_{i \in I}$ is a $\varepsilon$-g-Rieszbasis for $H$ with respect to $\left\{W_{i}\right\}_{i \in I}$, so this family is a $\mathrm{g}$-frame for $H$ with bounds $\frac{1}{1+\varepsilon}, 1+\varepsilon$ respectively. Hence $\frac{1}{1+\varepsilon} \leq\left\|S_{\Lambda}\right\| \leq(1+\varepsilon)$ and $\frac{1}{1+\varepsilon} \leq\left\|S_{\Lambda}^{-1}\right\| \leq(1+\varepsilon)$. On the other hand for any $f \in H$ and $n \in \mathrm{N}$ we have $\left\|S_{\Lambda}^{-1}\right\|^{-n}\|f\| \leq\left\|S_{\Lambda}^{n} f\right\| \leq\left\|S_{\Lambda}\right\|^{n}\|f\|$ which implies that $\left\|S_{\Lambda}^{-1}\right\|^{-n} I_{H} \leq S_{\Lambda}^{n} \leq\left\|S_{\Lambda}\right\|^{n} I_{H}$ consequently

$$
\frac{1}{(1+\varepsilon)^{n}} I_{H} \leq\left\|S_{\Lambda}^{-1}\right\|^{-n} I_{H} \leq S_{\Lambda}^{n} \leq\left\|S_{\Lambda}\right\|^{n} I_{H} \leq(1+\varepsilon)^{n} I_{H}
$$

This shows that

$\frac{1}{(1+\varepsilon)^{n}} I_{H} \leq S_{\Lambda}^{n} \leq(1+\varepsilon)^{n} I_{H}$ and so $\frac{1}{(1+\varepsilon)^{n}} I_{H} \leq S_{\Lambda}^{-n} \leq(1+\varepsilon)^{n} I_{H}$

Proposition 4.7 Let $\left\{\Lambda_{i}\right\}_{i \in I}$ be a $\varepsilon$-g-Riesz sequence for $H$ with respect to $\left\{W_{i}\right\}_{i \in I}$. Then $|\langle f, g\rangle| \leq 2 \varepsilon+\varepsilon^{2}$, for all partition $\left\{I_{1}, I_{2}\right\}$ of $I$ and $f \in \overline{\operatorname{span}}\left\{\Lambda_{\mathrm{l}}^{*}\left(W_{i}\right)\right\}_{i \in I_{1}}$, $g \in \overline{\operatorname{span}}\left\{\Lambda_{1}^{*}\left(W_{i}\right)\right\}_{i \in I_{2}}$, with $\|f\|=\|g\|=1$.

Proof. For all finite subsets $F_{1} \subseteq I_{1}, F_{2} \subseteq I_{2}$ and arbitrary vectors $g_{i} \in W_{i}\left(i \in F_{1} \cup F_{2}\right)$, suppose that $\varphi=\sum_{i \in F_{1}} \Lambda_{i}^{*}(g)_{i}$ and $\psi=\sum_{i \in F_{2}} \Lambda_{i}^{*}(g)_{i}$ with conditions $\|\varphi\|=\|\psi\|=1$. Then for any $|\lambda|=1$ we have

$$
\begin{aligned}
\operatorname{Re}(\langle\varphi, \lambda \psi\rangle) & =\frac{2 \operatorname{Re}(\langle\varphi, \lambda \psi\rangle)+2}{2}-1=\frac{\|\varphi+\lambda \psi\|^{2}}{2}-1 \\
& \leq \frac{1+\varepsilon}{2} \sum_{i \in F_{1} \cup F_{2}}\left\|g_{i}\right\|^{2}-1 \\
& =\frac{1+\varepsilon}{2}\left(\sum_{i \in F_{1}}\left\|g_{i}\right\|^{2}+\sum_{i \in F_{2}}\left\|g_{i}\right\|^{2}\right)-1 \\
& \leq \frac{(1+\varepsilon)^{2}}{2}\left(\|\varphi\|^{2}+\|\psi\|^{2}\right)-1=2 \varepsilon+\varepsilon^{2} .
\end{aligned}
$$

This yields $|\langle\varphi, \psi\rangle|=\max _{|\lambda|=1} \operatorname{Re}\langle\varphi, \lambda \psi\rangle \leq 2 \varepsilon+\varepsilon^{2}$, which implies that $|\langle f, g\rangle| \leq 2 \varepsilon+\varepsilon^{2}$.

Definition 4.8 For every $1 \leq i \leq M$, let $\Lambda_{i} \in \mathfrak{E}\left(H_{N}, W_{i}\right)$. Then we say that the family $\left\{\Lambda_{i}\right\}_{i=1}^{M}$ has the restricted isometry property with constant $0<\varepsilon<1$ for sets of size $s \leq N$, if for every $I \subseteq\{1,2, \ldots, M\}$ with $|I| \leq s$, the family $\left\{\Lambda_{i}\right\}_{i \in I}$ is a $\varepsilon$-g-Riesz sequence for $H_{N}$ with respect to $\left\{W_{i}\right\}_{i \in I}$. 
The next theorem is a generalization of Theorem $4.2^{11}$ to the g-frames situation.

THEOREM 4.9 Let $\left\{\Lambda_{i}\right\}^{M}$ be a tight g-frame for $H_{N}$ with respect to $\left\{W_{i}\right\}_{i=1}^{M}$ with the restricted isometry property with constant $0<\varepsilon<1$ for sets of size $s \leq N$. Suppose that $\left\{I_{j}\right\}_{j=1}^{L}$ is an arbitrary partition of $\{1,2, \ldots, M\}$ with $\left|I_{j}\right| \leq s$. Define

$V_{j}=\operatorname{span}\left\{\Lambda_{i}^{*}\left(W_{i}\right)\right\}_{i \in I_{j}}$ for all $1 \leq j \leq L$ then $\left\{V_{j}\right\}_{j=1}^{L}$ is a fusion frame for $H_{N}$ with fusion frame bounds $\frac{\sum_{i=1}^{M}\left\|\Lambda_{i}\right\|_{H S}^{2}}{(1+\varepsilon) N}$, $\frac{(1+\varepsilon) \sum_{i=1}^{M}\left\|\Lambda_{i}\right\|_{H S}^{2}}{N}$

and

$$
\frac{1}{(1+\varepsilon)} \sum_{i \in I_{j}}\left\|\Lambda_{i} f\right\|^{2} \leq\left\|\pi_{V_{j}} f\right\|^{2} \leq(1+\varepsilon) \sum_{i \in I_{j}}\left\|\Lambda_{i} f\right\|^{2}
$$

Proof. By the hypothesis $\left\{\Lambda_{i}\right\}_{i \in I_{i}}$ is a g-frame for $V_{j}$ with respect to $\left\{W_{i}\right\}_{i \in I_{j}}$ with g-frame bounds $\frac{1}{1+\varepsilon}, 1+\varepsilon$ respectively, for all $1 \leq j \leq L$. Let $S_{j}$ be g-frame operator of $\left\{\Lambda_{i}\right\}_{i \in I_{j}}$, which is a self-adjoint operator on $H_{N^{*}}$ Suppose that $\left\{e_{i}\right\}_{i=1}^{N}$ is an orthonormal basis for $S_{j}$, with eigenvalues $\left\{\lambda_{i}\right\}_{i=1}^{N}$, then $\lambda_{i}=0$ for all $\left|I_{j}\right|<i \leq N$ and $\frac{1}{1+\varepsilon} \leq \lambda_{1} \leq \lambda_{2} \leq \ldots \leq \lambda_{\left|I_{j}\right|} \leq 1+\varepsilon$. Since $\left\{e_{i}\right\}_{i=1}^{\left|I_{i}\right|}$ is an or thonormal basis for $V_{j}$, hence $\pi_{V_{j}} f=\sum_{i=1}^{\left|I_{j}\right|}\left\langle f, e_{i}\right\rangle e_{i}$, for any $f \in H_{N}$. We also have

$$
S_{j} f=S_{j}\left(\sum_{i=1}^{N}\left\langle f, e_{i}\right\rangle e_{i}\right)=\sum_{i=1}^{N}\left\langle f, e_{i}\right\rangle S_{j} e_{i}=\sum_{i=1}^{\left|I_{j}\right|}\left\langle f, e_{i}\right\rangle \lambda_{j} e_{i}
$$
have

which implies that $\left\langle S_{j} f, f\right\rangle=\sum_{i \in I_{j}} \lambda_{i}\left|\left\langle f, e_{i}\right\rangle\right|^{2}$. Thus we

$$
\begin{aligned}
\frac{1}{1+\varepsilon} \sum_{i \in I_{j}}\left\|\Lambda_{i} f\right\|^{2} & =\frac{1}{1+\varepsilon}\left\langle S_{j} f, f\right\rangle=\sum_{i \in I_{j}} \frac{\lambda_{i}}{1+\varepsilon}\left|\left\langle f, e_{i}\right\rangle\right|^{2} \\
& \leq\left\|\pi_{V_{j}}\right\|^{2} \leq(1+\varepsilon) \sum_{i \in I_{j}} \lambda_{i}\left|\left\langle f, e_{i}\right\rangle\right|^{2} \\
& =(1+\varepsilon)\left\langle S_{j} f, f\right\rangle=(1+\varepsilon) \sum_{i \in I_{j}}\left\|\Lambda_{i} f\right\|^{2}
\end{aligned}
$$

It follows that

$$
\frac{1}{1+\varepsilon} \sum_{j=1}^{L} \sum_{i \in I_{j}}\left\|\Lambda_{i} f\right\|^{2} \leq \sum_{j=1}^{L}\left\|\pi_{V_{j}} f\right\|^{2} \leq(1+\varepsilon) \sum_{j=1}^{L} \sum_{i \in I_{j}}\left\|\Lambda_{i} f\right\|^{2}
$$

Now by Proposition 4.1 we have

$$
\frac{\sum_{I=1}^{M}\left\|\Lambda_{i}\right\|_{H S}^{2}}{(1+\varepsilon) N}\|f\|^{2} \leq \sum_{j=1}^{L}\left\|\pi_{V j} f\right\|^{2} \leq \frac{(1+\varepsilon) \sum_{i=1}^{M}\left\|\Lambda_{i}\right\|_{H S}^{2}}{N}\|f\|^{2}
$$

COROLlary 4.10 Under the assumptions of Theorem 4.9 if $\{1,2, \ldots, L\} \subseteq\{1,2, \ldots, M\}$ and there exists a family $\left\{J_{j}\right\}_{j=1}^{L}$ such that $\sum_{j=1}^{L}\left|J_{j}\right| \leq s$ and $J_{j} \subseteq I_{j}$ for all $1 \leq j \leq L$. Then

$$
\begin{aligned}
\frac{1}{(1+\varepsilon)^{2}} \sum_{j=1}^{L}\left\|\sum_{i \in I_{j}} \Lambda_{i}^{*} g_{i}\right\|^{2} & \leq\left\|\sum_{j=1}^{L} \sum_{i \in I_{j}} \Lambda_{i}^{*} g_{i}\right\|^{2} \\
& \leq(1+\varepsilon)^{2} \sum_{j=1}^{L}\left\|\sum_{i \in I_{j}} \Lambda_{i}^{*} g_{i}\right\|^{2} .
\end{aligned}
$$

Proof. This follows from the Proposition 4.5.

The following theorem will give another method for obtaining a fusion frame from an unit norm tight frame for $H_{N}$ without having the restricted isometry property. Another form of this result can be found ${ }^{11}$ in Theorem 4.2.

TheOREM 4.11 Let $\left\{f_{i}\right\}_{i=1}^{M}$ be an unit norm tight frame of vectors for $H_{N}$ and let $\left\{I_{j}\right\}_{j=1}^{L}$ be a partition of $\{1,2, \ldots, M\}$. Define $W_{j}=\operatorname{span}\left\{f_{i}\right\}_{i \in I_{j}}$, then the family $\left\{W_{j}\right\}_{j=1}^{L}$ is a fusion frame for $H_{N}$ with fusion frame bounds $\frac{A M}{N}$ and $\frac{B M}{N}$ where $A=\min _{j=1}^{L} \min _{k=1}^{\operatorname{dim} W_{j}} \frac{1}{\lambda_{j k}}$, $B=\max _{j=1}^{L} \max _{k=1}^{\operatorname{dim} W_{j}} \frac{1}{\lambda_{j k}}$, and $\left\{\lambda_{i k}\right\}_{k=1}^{d i m W_{j}}$ is the family of eigenvalues of frame operator associated to $\left\{f_{i}\right\}_{i \in I_{j}}$.

Proof. Let $S_{j}$ be the frame operator associated to $\left\{f_{i}\right\}_{i \in I_{j}}$ and let $\left\{e_{j k}\right\}_{k=1}^{N}$ be the orthonormal basis for $H_{N}$ of eigenvectors of $S_{j}$ with eigenvalues $\left\{\lambda_{j k}\right\}_{k=1}^{N}$. Then $\lambda_{j k}=0$, for any $\operatorname{dim} W_{j}<k \leq N$ and $\left\{e_{j k}\right\}_{k=1}^{\operatorname{dim} W_{j}}$ is an orthonormal basis for $W_{j}$, which implies that $\left\langle S_{j} f, f\right\rangle=\sum_{k=1}^{\operatorname{dim} W_{j}} \lambda_{j k}\left|\left\langle f, e_{k}\right\rangle\right|^{2}$.

Now for any $f \in H_{N}$ we have

$$
\begin{gathered}
\min _{1 \leq k \leq \operatorname{dim} W_{j}} \frac{1}{\lambda_{j k}} \sum_{i \in I_{j}}\left|\left\langle f, f_{i}\right\rangle\right|^{2}=\min _{1 \leq k \leq \operatorname{dim} W_{j}} \frac{1}{\lambda_{j k}}\left\langle S_{j} f, f\right\rangle \\
=\sum_{k=1}^{\operatorname{dim} W_{j}} \frac{\lambda_{j k}}{\max _{1 \leq k \leq \operatorname{dim} W_{j}} \lambda_{j k}}\left|\left\langle f, e_{j k}\right\rangle\right|^{2}
\end{gathered}
$$




$$
\begin{aligned}
& \leq\left\|\pi_{W_{j}} f\right\|^{2} \leq \sum_{k=1}^{\operatorname{dim} W_{j}} \frac{\lambda_{j k}}{\min _{1 \leq k \leq \operatorname{dim} W_{j}} \lambda_{j k}}\left|\left\langle f, e_{j k}\right\rangle\right|^{2} \\
& =\max _{1 \leq k \leq \operatorname{dim} W_{j}} \frac{1}{\lambda_{j k}}\left\langle S_{j} f, f\right\rangle \\
& =\max _{1 \leq k \leq \operatorname{dim} W_{j}} \frac{1}{\lambda_{j k}} \sum_{i \in I_{j}}\left|\left\langle f, f_{i}\right\rangle\right|^{2}
\end{aligned}
$$

This yields

$$
\begin{aligned}
& \sum_{j=1}^{L} \sum_{i \in I_{j}} \min _{1 \leq l \leq \operatorname{dim} W_{j}} \frac{1}{\lambda_{j k}}\left|\left\langle f, f_{i}\right\rangle\right|^{2} \leq \sum_{j=1}^{L}\left\|\pi_{W_{j}} f\right\|^{2} \\
& \leq \sum_{j=1}^{L} \sum_{i \in I_{j}} \max _{1 \leq k \leq \operatorname{dim} W_{j}} \frac{1}{\lambda_{j k}}\left|\left\langle f, f_{i}\right\rangle\right|^{2} .
\end{aligned}
$$

Put $A=\min _{j=1}^{L} \min _{k=1}^{\operatorname{dim} W_{j}} \frac{1}{\lambda_{j k}}, \quad B=\max _{j=1}^{L} \max _{k=1}^{\operatorname{dim} W_{j}} \frac{1}{\lambda_{j k}}$.

Then

$$
\frac{A M}{N}\|f\|^{2} \leq \sum_{i=1}^{L}\left\|\pi_{W_{j}} f\right\|^{2} \leq \frac{B M}{N}\|f\|^{2} .
$$

The next corollary generalizes Theorem 4.11 to the g-frames situation which the proof leaves to interested readers.

COROLLARY 4.12 Let $\left\{\Lambda_{i}\right\}_{i=1}^{M}$ be a tight g-frame for $H_{N}$ with respect to $\left\{W_{i}\right\}_{i=1}^{M}$ and let $\left\{I_{j}\right\}_{j=1}^{L}$ be a partition of $\{1,2, \ldots, M\}$. Define $V_{j}=\operatorname{span}\left\{\Lambda_{i}^{*}\left(W_{i}\right)\right\}_{i \in I_{i}}$, then the family $\left\{V_{j}\right\}_{j=1}^{L}$ is a fusion frame for $H_{N}$ with fusion frame bounds $\frac{A \sum_{i=1}^{M}\left\|\Lambda_{i}\right\|_{H S}^{2}}{N}$ and $\quad \frac{B \sum_{i=1}^{M}\left\|\Lambda_{i}\right\|_{H S}^{2}}{N} \quad$ where $\quad A=\min _{j=1}^{L} \min _{k=1}^{\operatorname{dim} W_{j}} \frac{1}{\lambda_{j k}}$, $B=\max _{j=1}^{L} \max _{k=1}^{\operatorname{dim} W_{j}} \frac{1}{\lambda_{j k}}$, and $\left\{\lambda_{j k}\right\}_{k=1}^{\operatorname{dim} W_{j}}$ is the family of eigenvalues of $g$-frame operator associated to $\left\{\Lambda_{i}\right\}_{i \in I_{j}}$.

\section{Stability of g-frames under Erasure of Operators}

Our purpose of this section is to study the conditions which under removing some element from a g-frame, again we obtain another g-frame. The next theorem gives a erasure result of $g$-frames so that Theorem 4.3 obtained $^{8}$ is a special case of it.

TheOrem 5.1 Let $\Lambda=\left\{\Lambda_{i}\right\}_{i \in I}$ be a g-frame for $H$ with respect to $\left\{W_{i}\right\}_{i \in I}$ with g-frame bounds $A$ and $B$ and let
$J \subseteq I$. Then $\left\{\Lambda_{i}\right\}_{i \in I-J}$ is a g-frame for $H$ with respect to $\left\{W_{i}\right\}_{i \in I-J}$ with bounds $\frac{A^{2}}{B}\left\|\left(I_{H}-\sum_{i \in J} S_{\Lambda}^{-1} \Lambda_{i}^{*} \Lambda_{i}\right)^{-1}\right\|^{-2}$ and $B$ if and only if $I_{H}-\sum_{i \in J} S_{\Lambda}^{-1} \Lambda_{i}^{*} \Lambda_{i}$ be a bounded invertible operator on $H$.

Proof. Since $\Lambda=\left\{\Lambda_{i}\right\}_{i \in I}$ is a g-frame for $H$ with respect to $\left\{W_{i}\right\}_{i \in I}$ thus for any $f \in H$ we have

$$
f=\sum_{i \in I} S_{\Lambda}^{-1} \Lambda_{i}^{*} \Lambda_{i} f=\sum_{i \in J} S_{\Lambda}^{-1} \Lambda_{i}^{*} \Lambda_{i} f+\sum_{i \in I-J} S_{\Lambda}^{-1} \Lambda_{i}^{*} \Lambda_{i} f .
$$
have

Thus $I_{H}-\sum_{i \in J} S_{\Lambda}^{-1} \Lambda_{i}^{*} \Lambda_{i}=\sum_{i \in I-J} S_{\Lambda}^{-1} \Lambda_{i}^{*} \Lambda_{i} . \quad$ Moreover we

$$
\begin{aligned}
\left\|I_{H}-\sum_{i \in J} S_{\Lambda}^{-1} \Lambda_{i}^{*} \Lambda_{i} f\right\| & =\left\|\sum_{i \in I-J} S_{\Lambda}^{-1} \Lambda_{i}^{*} \Lambda_{i} f\right\| \\
& =\sup _{\|g\|=1}\left|\left\langle\sum_{i \in I-J} S_{\Lambda}^{-1} \Lambda_{i}^{*} \Lambda_{i} f, g\right\rangle\right| \\
& =\sup _{\|g\|=1}\left|\left\langle\sum_{i \in I-J} \Lambda_{i} f, \Lambda_{i} S_{\Lambda}^{-1} g\right\rangle\right| \\
& \leq \sup _{\|g\|=1} \sum_{i \in I-J}\left\|\Lambda_{i} f\right\|\left\|\Lambda_{i} S_{\Lambda}^{-1} g\right\| \\
& \leq \sup _{\|g\|=1}\left(\sum_{i \in I-J}\left\|\Lambda_{i} f\right\|^{2}\right)^{\frac{1}{2}}\left(\sum_{i \in I-J}\left\|\Lambda_{i} S_{\Lambda}^{-1} g\right\|^{2}\right)^{\frac{1}{2}} \\
& \leq \sup _{\|g\|=1} \sqrt{B}\left\|S_{\Lambda}^{-1} g\right\|\left(\sum_{i \in I-J}\left\|\Lambda_{i} f\right\|^{2}\right)^{\frac{1}{2}} \\
& \leq \frac{\sqrt{B}}{A}\left(\sum_{i \in I-J}\left\|\Lambda_{i} f\right\|^{2}\right)^{\frac{1}{2}}
\end{aligned}
$$

Now, if $I_{H}-\sum_{i \in j} S_{\Lambda}^{-1} \Lambda_{i}^{*} \Lambda_{i}$ is invertible on $H$. Then

$$
\begin{aligned}
\frac{A^{2}}{B}\left\|\left(I_{H}-\sum_{i \in J} S_{\Lambda}^{-1} \Lambda_{i}^{*} \Lambda_{i}\right)^{-1}\right\|^{-2}\|f\|^{2} & \leq \frac{A^{2}}{B}\left\|\left(I_{H}-\sum_{i \in J} S_{\Lambda}^{-1} \Lambda_{i}^{*} \Lambda_{i} f\right)\right\|^{2} \\
& \leq \sum_{i \in I-J}\left\|\Lambda_{i} f\right\|^{2}
\end{aligned}
$$

On the other hand, since $\Lambda$ is a g-frame hence $\left\{\Lambda_{i}\right\}_{i \in I-J}$ is a g-Bessel sequence, which shows that $\left\{\Lambda_{i}\right\}_{i \in I-J}$ is a g-frame. Conversively, suppose that $\left\{\Lambda_{i}\right\}_{i \in I-J}$ is a g-frame for $\mathrm{H}$ with respect to $\left\{W_{i}\right\}_{i \in I-J}$, with g-frame bounds $A$ and $B$. We first show that $I_{H}-\sum_{i \in J} S_{\Lambda}^{-1} \Lambda_{i}^{*} \Lambda_{i}$ is injective. Let $\left(I_{H}-\sum_{i \in J} S_{\Lambda}^{-1} \Lambda_{i}^{*} \Lambda_{i}\right) f=0$, then $\sum_{i \in I-J} S_{\Lambda}^{-1} \Lambda_{i}^{*} \Lambda_{i} f=0$ hence $\sum_{i \in I-J} \Lambda_{i}^{*} \Lambda_{i} f=0$. It follows that 


$$
\begin{aligned}
A\|f\|^{2} \leq \sum_{i \in I-J}\left\|\Lambda_{i} f\right\|^{2} & =\sum_{i \in I-J}\left\langle\Lambda_{i} f, \Lambda_{i} f\right\rangle \\
& =\left\langle\sum_{i \in I-J} \Lambda_{i}^{*} \Lambda_{i} f, f\right\rangle=0
\end{aligned}
$$

which implies that $f=0$. Moreover, if $\left(I_{H}-\sum_{i \in J} S_{\Lambda}^{-1} \Lambda_{i}^{*} \Lambda_{i}\right)^{*} f=0$ then $\sum_{i \in I-J} \Lambda_{i}^{*} \Lambda_{i} S_{\Lambda}^{-1} f=0$ and therefore $S_{\Lambda}^{-1} f=0$, it follows that $f=0$. This finishes the proof.

Corollary 5.2 Let $\left\{\Lambda_{i}\right\}_{i \in I}$ be a g-frame for $H$ with respect to $\left\{W_{i}\right\}_{i \in I}$ and let $J \subseteq I$. If there exists $0 \neq f_{0} \in H$ such that $\sum_{i \in J} S_{\Lambda}^{-1} \Lambda_{i}^{*} \Lambda_{i} f_{0}=f_{0}$ Then $\left\{\Lambda_{i}\right\}_{i \in I-J}$ is not a g-frame for $H$.

Proof. If there exists $0 \neq f_{0} \in H$ such that $\sum_{i \in I} S_{\Lambda}^{-1} \Lambda_{i}^{*} \Lambda_{i} f_{0}=f_{0}$

Then $\sum_{i \in I-J} S_{\Lambda}^{-1} \Lambda_{i}^{*} \Lambda_{i} f_{0}=0$, hence $\sum_{i \in I-J} \Lambda_{i}^{*} \Lambda f_{0}=0$. It follows that

$$
\begin{aligned}
\sum_{i \in I-J}\left\|\Lambda_{i} f_{0}\right\|^{2} & =\sum_{i \in I-J}\left\langle\Lambda_{i} f_{0}, \Lambda_{i} f_{0}\right\rangle \\
& =\left\langle\sum_{i \in I-J} S_{\Lambda}^{-1} \Lambda_{i}^{*} \Lambda_{i} f_{0}, f_{0}\right\rangle=0 .
\end{aligned}
$$

Therefore, $\left\{\Lambda_{i}\right\}_{i \in I-J}$ is not a g-frame.

Corollary 5.3 Let $\left\{\Lambda_{i}\right\}_{i \in I}$ be a $A$-tight g-frame for $H$ with respect to $\left\{W_{i}\right\}_{i \in I}$ and let $J \subseteq I$. If there exists $0 \neq f_{0} \in H$ such that $\sum_{i \in J} \Lambda_{\mathrm{i}}^{*} \Lambda_{i} f_{0}=A f_{0}$, then $\left\{\Lambda_{i}\right\}_{i \in I}$ is not a g-frame for $H$.

\section{Acknowledgement}

We thank an anonymous referee for constructive comments which helped to improve the paper.

\section{References}

1. Duffin RJ, Schaeffer AC. A class of nonharmonic Fourier series. Trans Am Math Soc.1952; 72(2):341-66.

2. Daubechies I, Grossmann A, Meyer Y. Painless nonorthogonal expansions. J Math Phys. 1986; 27(5):1271-83.

3. Casazza PG, Kutyniok G. Frames of subspaces. in Wavelets, Frames and Operator Theory (College Park, MD, 2003), Contemp Math Amer Math Soc Providence, RI. 2004; 345:87-113.

4. Sun W. G-frames and G-Riesz bases. J Math Anal Appl. 2006; 322(1); 437-52.

5. Christensen O. An introduction to frames and Riesz bases. Boston: Birkhauser; 2003.

6. Christensions O, Kim HO, Kim RY. Extensions of Bessel sequences to dual pairs of frames. Applied and Computational Harmonic Analysis. 2013 Mar; 34(2): 224-233.

7. Asgari MS, Khosravi A. Frames and bases of subspaces in Hilbert spaces. J Math Anal Appl. 2005; 308(2):541-53.

8. Asgari MS. On the stability of Fusion Frames(Frames of Subspaces). Acta Math Sci Ser B. 2011; 31(4):1633-42.

9. Abdollahpour MR, Najati A. Approximation of the inverse g-frameoperator.Proceedings-MathematicalSciences.2011; 121(2):143-54.

10. Li DF, Sun W. Expansion of frames to tight frames. Acta Math Sin (Engl Ser). 2009; 25(2):287-292.

11. Bodmann BG, Cahill J, Casazza PG. Fusion frames and the restricted isometry property. Num Func Anal Optim. 2012; 33(7-9):770-90. 